\title{
A Stern-Gerlach separator of chiral enantiomers based on the Casimir-Polder potential
}

\author{
Fumika Suzuki, ${ }^{1,2,3}$, 用 Takamasa Momose,, , 用 and S. Y. Buhmann ${ }^{3,4}$, 用 \\ ${ }^{1}$ Department of Chemistry, University of British Columbia, Vancouver, British Columbia, Canada V6T 1Z1 \\ ${ }^{2}$ Department of Physics and Astronomy, University of British Columbia, Vancouver, British Columbia, Canada V6T 1Z1 \\ ${ }^{3}$ Physikalisches Institut, Albert-Ludwigs-Universität Freiburg, \\ Hermann-Herder-Str. 3, 79104 Freiburg, Germany \\ ${ }^{4}$ Freiburg Institute for Advanced Studies, Albert-Ludwigs-Universität Freiburg, Albertstr. 19, 79104 Freiburg, Germany
}

(Dated: December 19, 2018)

\begin{abstract}
We propose a method to separate enantiomers using parity violation in the Casimir-Polder potential between chiral mirrors and chiral molecules. The proposed setup involves a molecular beam composed of chiral molecules passing through a planar cavity consisting of two chiral mirrors. Enantiomers of opposite handedness are deflected differently due to a chiral dependence of the CasimirPolder potential resulting in the separation of the enantiomers. Our setup provides an alternative experimental tool for enantiomer separation, as well as shedding light on the fundamental properties of the Casimir-Polder potential.
\end{abstract}

\section{INTRODUCTION}

Many molecules are chiral which can exist in left- and right-handed forms (i.e., non-superimposable mirror images). These two forms of a chiral molecule are known as enantiomers. Distinguishing two types of enantiomers is of great practical importance. For example, in designing pharmaceuticals, it is necessary to choose the right enantiomer to obtain the desired effects since the other enantiomer is less active, inactive, or can even have adverse side effects, including high toxicity. Therefore, developing technology which separates enantiomers can improve pharmacy by introducing medicines composed of only one enantiomer which enhances the desired effects, while eliminating the side effects [1].

Common methods for chiral separation include chromatography [2] and crystalization [3]. Chiral separations using chromatography now represent a popular, robust routine technique utilized in laboratories. However, the selection of columns still remains a matter of trial and error, and it is difficult to find materials that show both high efficiency and high enantio-selectivity. SternGerlach type separators for enantiomers have been proposed that are based on inhomogeneous laser fields [4, 5]. The trajectories of the emerging molecular beams depend on both spin and handedness. In such schemes, the orientation and rotation of the molecules needs to be carefully addressed 6].

Here we propose a method for the separation of chiral enantiomers that is based on the Casimir-Polder (CP) potential. CP forces are effective quantum electrodynamical forces between neutral, unpolarised molecules and macroscopic bodies which arise from the interaction of the objects' charge and current densities with

\footnotetext{
* fumika@chem.ubc.ca

$\dagger$ momose@chem.ubc.ca

$\ddagger$ stefan.buhmann@physik.uni-freiburg.de
}

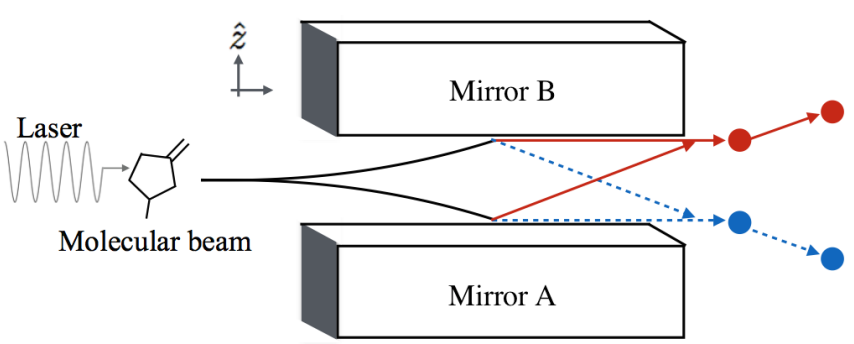

FIG. 1: (Color online) A beam of chiral molecules driven by a laser field passes through a planar cavity consisting of two chiral mirrors. Enantiomers of opposite handedness are deflected differently due to the chiral component of the $\mathrm{CP}$ potential.

the vacuum electromagnetic field. Originally predicted by Casimir and Polder, they are typically attractive and purely monotonic for ground-state molecules and bodies with a purely electric response [7]. It was later seen that both the direction and distance dependence of the potential changes when considering excited molecules. Here, the possibility that the molecule emits a real photon which gets reflected off the body and subsequently reabsorbed leads to a potential whose sign depends on the relative phase of emitted and reabsorbed photons [8, 9. Depending on the detuning between the radiation emitted by the molecule and the plasmonic resonances of the body, the potential can be attractive or repulsive in the near zone [10] while oscillating with distance in the far zone [11; and the potential may strongly exceed its ground-state counterpart.

In addition, the sign of the CP force may depend on the electromagnetic response of the interacting objects. Motivated by similar findings for the Casimir force by Boyer [12, it was found that $\mathrm{CP}$ forces due to interactions of electric atoms with magnetic surfaces - or vice versa - are repulsive [13 15] in contrast to the attractive electric-electric force. Similar repulsive forces have been 
predicted for circularly polarised atoms interacting with axionic topological insulators 16 and-most crucially for this work - for chiral molecules interacting with chiral surfaces. Here the forces are attractive for objects of the same handedness and repulsive for opposite handedness [17.

The chiral component of the CP potential is hence sensitive to the handedness of molecules and can be attractive or repulsive depending on their chirality. This is because the $\mathrm{CP}$ potential between a chiral molecule and a chiral mirror depends on the optical rotatory strength of the molecule which is a pseudoscalar that changes sign under a parity inversion [18]. The potential could hence be used to differentiate enantiomers of opposite handedness. Using this property of the CP potential, it would be possible to identify the handedness of the chiral molecule by setting up a Stern-Gerlach type discriminator for enantiomers. Although the electric component of the CP force has been already observed [19, here we aim to measure its chiral component which has not yet been observed.

The closely related chiral van der Waal force between two molecules has been discussed earlier, where again, enantiomer-discriminatory interactions are predicted when both particles are chiral 20 23. Since these forces are very weak, a stronger force between a chiral molecule and an achiral molecule has been proposed which is mediated by a nearby chiral surface [24.

Our proposed setup uses the molecular beam deflection technique. We let a molecular beam composed of chiral molecules pass through a planar cavity consisting of two chiral mirrors (FIG. 1). Although the electric component of the CP force between the cavity and the molecules acts on both enantiomers in the same way, the chiral component can be attractive or repulsive depending on the handedness of the molecules and can deflect enantiomers of opposite handedness differently and separate them. To exploit the enhanced excited-state CP force, the molecules are subject to a weakly detuned driving laser while passing through the cavity. The method proposed here is more universal than the above-mentioned conventional schemes, since we only utilize the interaction between a chiral mirror and chiral molecules induced by photo excitations and emissions. All molecules have characteristic excitation spectra, and therefore a suitable, resonant light source (either UV or IR) is the only prerequisite for the present method. The same chiral mirror can be used for enantiomer separation of a wide range of molecules.

The article is organized as follows: In Sec. II we study the behavior of the CP potential between the cavity and the chiral molecule driven by a laser field within the framework of macroscopic quantum electrodynamics. We compare the CP potential experienced by 3-methylcyclopentanone (3-MCP) molecules with electric circular dichroism (ECD) and propylene-oxide molecules with vibrational circular dichroism (VCD). We find that the $\mathrm{CP}$ potential for propylene-oxide molecules with VCD can
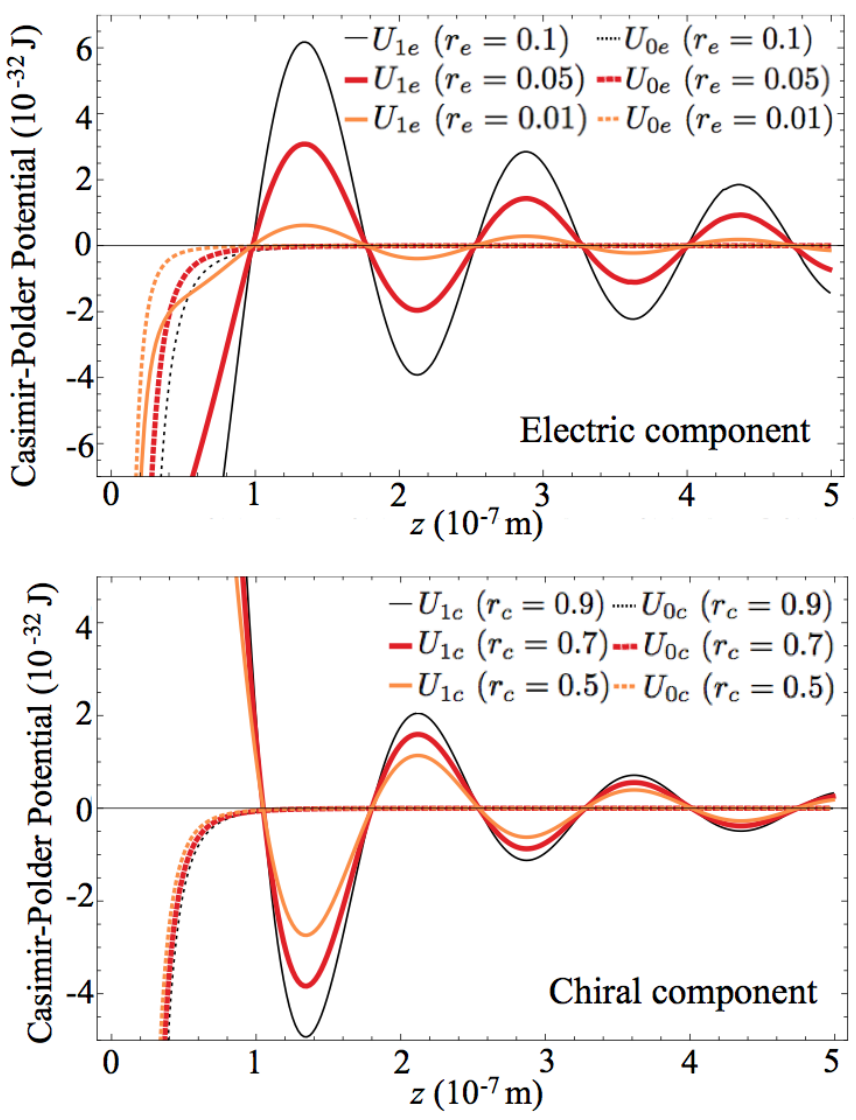

FIG. 2: (Color online) The electric and chiral component (experienced by the 3-MCP molecule with the positive $R_{01}$ ) of the $\mathrm{CP}$ potential with different reflection coefficients: $r_{e}=0.01,0.05$ and $0.1, r_{c}=0.5$, 0.7 and 0.9 . The solid lines: the excited-state potential, the dotted lines: the ground-state potential.

depend on temperature. We also observe the enhancement of the CP potential due to the cavity structure when the cavity is small enough that it contains only a few molecular transition wavelengths. However, experimentally we use a realistic setup where the cavity width is $1 \mathrm{~mm}$ and such enhancement can not be observed. In Sec. III] we simulate separation of enantiomers using our setup by studying trajectories of the molecules in the cavity. Since the strength of the CP potential depends on the frequency of photons and the electric and chiral components depend on the electric dipole strength and the optical rotatory strength of the molecules respectively, we use chiral molecules with ECD whose optical rotatory strength is large compared to its electric dipole moment in the simulation. In this article, we use 3-methylcyclopentanone as an example for a quantitative analysis. Finally we conclude that our proposed setup can be used for separating enantiomers of opposite handedness and detection of the chiral dependence of the $\mathrm{CP}$ potential within reach of current technology (Sec. IV). 

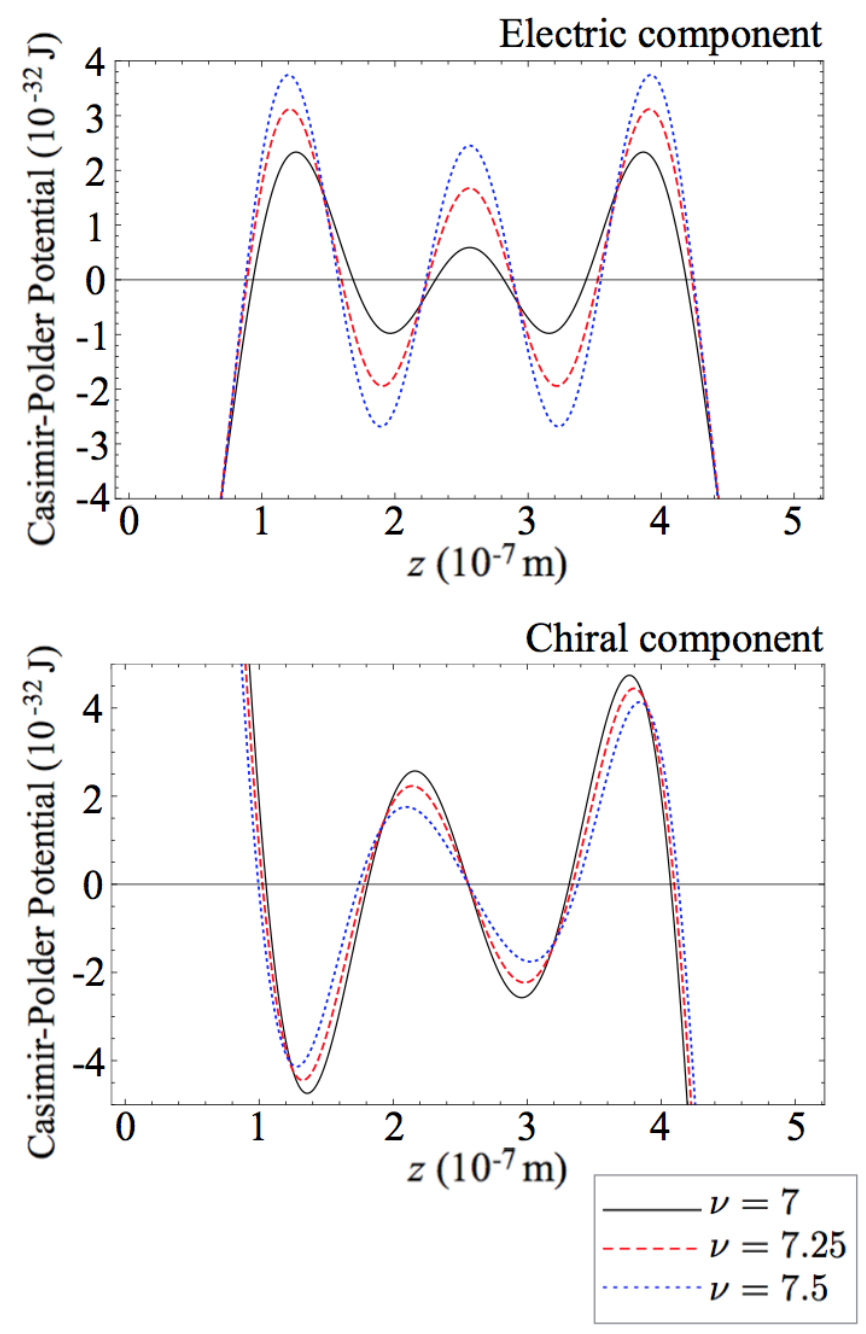

FIG. 3: (Color online) The enhancement of the chiral component occurs when $a=\nu \lambda_{10} / 4$ and $\nu$ is odd natural number if the two mirrors have opposite chirality and the cavity width contains only a few molecular transition wavelengths. $r_{e}=0.05, r_{c}=0.8$.

\section{BEHAVIOR OF THE CASIMIR-POLDER POTENTIAL}

Within the framework of macroscopic quantum electrodynamics [25, 26], the CP potential $U_{C P}$ between the cavity and the chiral molecule (approximated as two-level system) driven by a laser field is given by [27, 28]

$$
U_{C P}=p_{0}(t) U_{0}+p_{1}(t) U_{1}
$$

where the populations of the ground state $p_{0}(t)$ and that of the excited state $p_{1}(t)$ are written as

$$
\begin{aligned}
& p_{0}(t)=\frac{\Omega^{2}}{\Delta^{2}+\Omega^{2}} \cos ^{2}\left(\frac{1}{2} \sqrt{\Delta^{2}+\Omega^{2}} t\right)+\frac{\Delta^{2}}{\Delta^{2}+\Omega^{2}}, \\
& p_{1}(t)=\frac{\Omega^{2}}{\Delta^{2}+\Omega^{2}} \sin ^{2}\left(\frac{1}{2} \sqrt{\Delta^{2}+\Omega^{2}} t\right) .
\end{aligned}
$$

Note that the coherence of the two states gives rise to an additional term which oscillates with optical frequencies and is hence unobservable. Here $\Omega$ is the Rabi frequency and the detuning $\Delta=\omega_{L}-\omega_{10} \cdot \omega_{10}$ is the molecular transition frequency and $\omega_{L}$ is the driven frequency of the laser. We choose $\omega_{L} \approx \omega_{10}$ to ensure a large excitedstate population. The potential for the molecule in the ground state is $U_{0}=U_{0 e}+U_{0 c}$, and that for the molecule in the excited state is $U_{1}=U_{1 e}+U_{1 c}$, where the electric components of the potential are given by

$$
\begin{aligned}
U_{0 e}= & \frac{\hbar \mu_{0}}{2 \pi} \int_{0}^{\infty} d \xi \xi^{2} \alpha(i \xi) \operatorname{tr} \mathbf{G}(\mathbf{r}, \mathbf{r}, i \xi), \\
U_{1 e}= & -\frac{\hbar \mu_{0}}{2 \pi} \int_{0}^{\infty} d \xi \xi^{2} \alpha(i \xi) \operatorname{tr} \mathbf{G}(\mathbf{r}, \mathbf{r}, i \xi) \\
& -\frac{\mu_{0}}{3} \omega_{10}^{2}\left|\mathbf{d}_{01}\right|^{2} \operatorname{tr}\left[\operatorname{Re} \mathbf{G}\left(\mathbf{r}, \mathbf{r}, \omega_{10}\right)\right],
\end{aligned}
$$

while its chiral components are given by 17 .

$$
\begin{aligned}
U_{0 c}= & -\frac{\hbar \mu_{0}}{\pi} \int_{0}^{\infty} d \xi \xi \Gamma(i \xi) \operatorname{tr}[\nabla \times \mathbf{G}(\mathbf{r}, \mathbf{r}, i \xi)], \\
U_{1 c}= & \frac{\hbar \mu_{0}}{\pi} \int_{0}^{\infty} d \xi \xi \Gamma(i \xi) \operatorname{tr}[\nabla \times \mathbf{G}(\mathbf{r}, \mathbf{r}, i \xi)] \\
& +\frac{2 \mu_{0} \omega_{10} R_{01}}{3} \operatorname{tr}\left[\nabla \times \operatorname{Re}\left[\mathbf{G}\left(\mathbf{r}, \mathbf{r}, \omega_{10}\right)\right]\right],
\end{aligned}
$$

and

$$
\alpha(i \xi)=\frac{2}{3 \hbar} \frac{\omega_{10}\left|\mathbf{d}_{01}\right|^{2}}{\omega_{10}^{2}+\xi^{2}}, \quad \Gamma(i \xi)=-\frac{2}{3 \hbar} \frac{\xi R_{01}}{\omega_{10}^{2}+\xi^{2}}
$$

Here $\hbar$ is Planck's constant, $\mu_{0}$ is vacuum permeability, $\mathbf{d}_{01}$ is the electric dipole transition matrix element, and the optical rotatory strength $R_{01}=\operatorname{Im}\left(\mathbf{d}_{01} \cdot \mathbf{m}_{10}\right)$ where $\mathbf{m}_{10}$ is the corresponding magnetic dipole moment matrix element. We have ignored the magnetic component of the potential which is a factor of $\left(\left|\mathbf{m}_{10}\right| /\left|\mathbf{d}_{01}\right|\right)^{2} \sim \alpha_{f}^{2}$ smaller than the electric component, and is a factor of $\left|\mathbf{m}_{10}\right| /\left|\mathbf{d}_{01}\right| \sim \alpha_{f}$ smaller than the chiral component, with $\alpha_{f}$ being the fine-structure constant. Since the optical rotatory strengths of enantiomers are generally identical in magnitude but opposite in sign, the chiral components of the $\mathrm{CP}$ potential (4) take opposite signs for enantiomers.

Note that, when the average thermal photon number

$$
n\left(\omega_{10}\right)=\frac{1}{e^{\hbar \omega_{10} /\left(k_{B} T\right)}-1}
$$

is not negligible, (3) and (4) can be replaced by the ther- 
mal CP potentials which are written as [26, 29, 31

$$
\begin{aligned}
U_{0 e}^{\text {therm }}= & \mu_{0} k_{B} T \sum_{j=0}^{\infty}\left(1-\frac{1}{2} \delta_{j 0}\right) \xi_{j}^{\prime 2} \alpha\left(i \xi_{j}^{\prime}\right) \operatorname{tr} \mathbf{G}\left(\mathbf{r}, \mathbf{r}, i \xi_{j}^{\prime}\right) \\
& +\frac{\mu_{0}}{3} n\left(\omega_{10}\right) \omega_{10}^{2}\left|\mathbf{d}_{01}\right|^{2} \operatorname{tr}\left[\operatorname{Re} \mathbf{G}\left(\mathbf{r}, \mathbf{r}, \omega_{10}\right)\right], \\
U_{1 e}^{\text {therm }}= & -U_{0 e}^{\text {therm }} \\
& -\frac{\mu_{0}}{3}\left[n\left(\omega_{10}\right)+1\right] \omega_{10}^{2}\left|\mathbf{d}_{01}\right|^{2} \operatorname{tr}\left[\operatorname{Re} \mathbf{G}\left(\mathbf{r}, \mathbf{r}, \omega_{10}\right)\right], \\
U_{0 c}^{\text {therm }}= & -2 \mu_{0} k_{B} T \sum_{j=0}^{\infty}\left(1-\frac{1}{2} \delta_{j 0}\right) \xi_{j}^{\prime} \Gamma\left(i \xi_{j}^{\prime}\right) \\
& \times \operatorname{tr}[\nabla \times \mathbf{G}(\mathbf{r}, \mathbf{r}, i \xi)] \\
& -\frac{2 \mu_{0} \omega_{10} R_{01}}{3} n\left(\omega_{10}\right) \operatorname{tr}\left[\nabla \times \operatorname{Re}\left[\mathbf{G}\left(\mathbf{r}, \mathbf{r}, \omega_{10}\right)\right],\right. \\
= & -U_{0 c}^{\text {therm }} \\
& +\frac{2 \mu_{0} \omega_{10} R_{01}}{3}\left[n\left(\omega_{10}\right)+1\right] \operatorname{tr}\left[\nabla \times \operatorname{Re}\left[\mathbf{G}\left(\mathbf{r}, \mathbf{r}, \omega_{10}\right)\right]\right], \\
U_{1 c}^{\text {therm }} &
\end{aligned}
$$

where $\xi_{j}^{\prime}=2 \pi k_{B} T j / \hbar(j=0,1,2, \ldots)$ are Matsubara frequencies 32 . The thermal $\mathrm{CP}$ potentials have contributions proportional to $n\left(\omega_{10}\right)$ and $n\left(\omega_{10}\right)+1$ due to the absorption and emission of photons by the molecule respectively. Different from (3) and (4), we see that a ground-state molecule also exhibits a resonant contribution associated with absorption of thermal photons.

The scattering Green's tensor $\mathbf{G}(\mathbf{r}, \mathbf{r}, \omega)$ for our cavity setup can be written as 33

$$
\begin{aligned}
& \mathbf{G}(\mathbf{r}, \mathbf{r}, \omega)=\frac{i}{8 \pi^{2}} \int \frac{d^{2} k^{\|}}{k^{\perp}} \\
& \times\left[\sum_{\sigma_{1} \sigma_{2}=s, p} e^{2 i k^{\perp} z}\left(\mathbf{D}^{-1} \cdot \mathbf{R}\right)^{\left(\sigma_{1}, \sigma_{2}\right)} \mathbf{e}_{\sigma_{1}}\left(k^{\perp}\right) \mathbf{e}_{\sigma_{2}}\left(-k^{\perp}\right)\right. \\
& \left.+\sum_{\sigma_{1} \sigma_{2}=s, p} e^{2 i k^{\perp}(a-z)}\left(\mathbf{R}^{\prime} \cdot \mathbf{D}^{-1}\right)^{\left(\sigma_{1}, \sigma_{2}\right)} \mathbf{e}_{\sigma_{1}}\left(-k^{\perp}\right) \mathbf{e}_{\sigma_{2}}\left(k^{\perp}\right)\right]
\end{aligned}
$$

where the polarization sum runs over $s-$ and $p$-polarizations, $\mathbf{D}=\mathbf{I}-e^{2 i a k^{\perp}} \mathbf{R} \cdot \mathbf{R}^{\prime}, k^{\|}$and $k^{\perp}$ are the components of the wave vector parallel and perpendicular to the surface of mirrors respectively and $k^{\perp 2}=\omega^{2} / c^{2}-k^{\| 2}, a$ is the distance between two mirrors (i.e., the width of the cavity). We discarded terms that are independent of the position of the molecule $z$ $(0<z<a)$. Mirror $\mathrm{A}$ is located at $z=0$ while mirror $\mathrm{B}$ is located at $z=a . \quad \mathbf{R}=\left(\begin{array}{ll}r_{s s} & r_{s p} \\ r_{p s} & r_{p p}\end{array}\right)=\left(\begin{array}{ll}-r_{e} & r_{c} \\ -r_{c} & r_{e}\end{array}\right)$ and $\mathbf{R}^{\prime}=\left(\begin{array}{ll}r_{s s}^{\prime} & r_{s p}^{\prime} \\ r_{p s}^{\prime} & r_{p p}^{\prime}\end{array}\right)=\left(\begin{array}{cc}-r_{e} & -r_{c} \\ r_{c} & r_{e}\end{array}\right)$ are the reflection matrices of mirrors $\mathrm{A}$ and $\mathrm{B}$, respectively, where the superscript ${ }^{\left(\sigma_{1} \sigma_{2}\right)}$ represents the element $\left(\sigma_{1} \sigma_{2}\right)$ of the matrix. The mirrors are assumed to have opposite chirality. We adopt the convention that a left-handed mirror or a mirror of positive chirality rotates the polarisation of
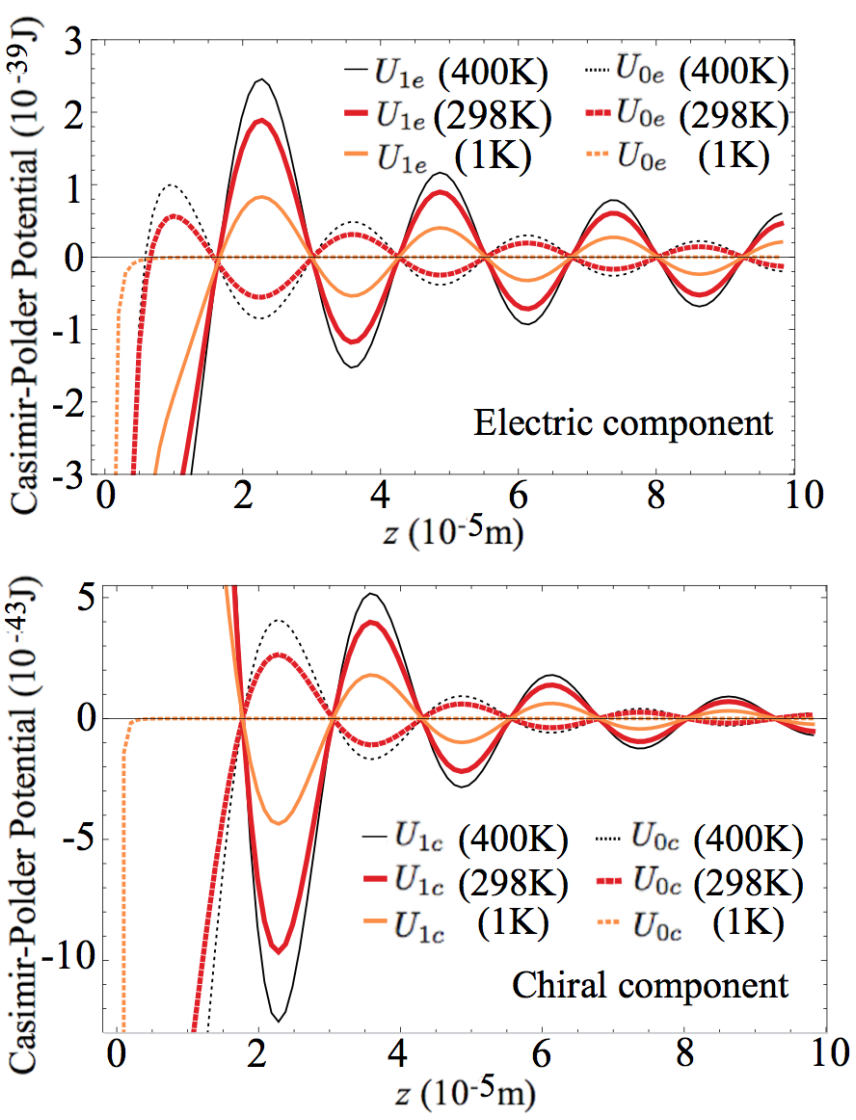

FIG. 4: (Color online) The electric and chiral component (experienced by the $(R)$-propylene-oxide molecule with the positive $R_{01}$ ) of the CP potential with different temperatures. $r_{e}=0.05, r_{c}=0.8$. The solid lines: the excited-state potential, the dotted lines: the ground-state potential.

an electromagnetic wave in a counter-clockwise direction upon reflection when travelling alongside the wave, while a right-handed mirror of negative chirality rotates it in a clockwise direction. With this convention, $r_{c}>0$ implies that mirror $\mathrm{A}$ is right-handed or of negative chirality while mirror B is left-handed or of positive chirality. Note that we are neglecting the dependence of the reflection coefficients on frequency and on the in-plane component of the wave vector $\mathbf{k}^{\|}$. This is justified as we are mainly interested in the dominant resonant part of the CasimirPolder potential which is governed by a single transition frequency and travelling-wave contributions at normal incidence. In practice, we will obtain the entries of the reflection matrix from laser reflection experiments on chiral materials currently under development.

In the case of linearly polarized light, the $p$ - and $s$ - polarized waves $\mathbf{E}_{s}, \mathbf{E}_{p}$ are reflected as $\mathbf{E}_{s} \rightarrow r_{s p} \mathbf{E}_{p}+r_{s s} \mathbf{E}_{s}$ and $\mathbf{E}_{p} \rightarrow r_{p p} \mathbf{E}_{p}+r_{p s} \mathbf{E}_{s}$ by the chiral mirror respectively. Normal mirrors have small or zero values for $r_{s p}$ and $r_{p s}$. Perfect chiral mirrors have $\left|r_{s p}\right|=\left|r_{p s}\right|=1$ and $\left|r_{s s}\right|=\left|r_{p p}\right|=0$, which exhibits the strongest chiral de- 


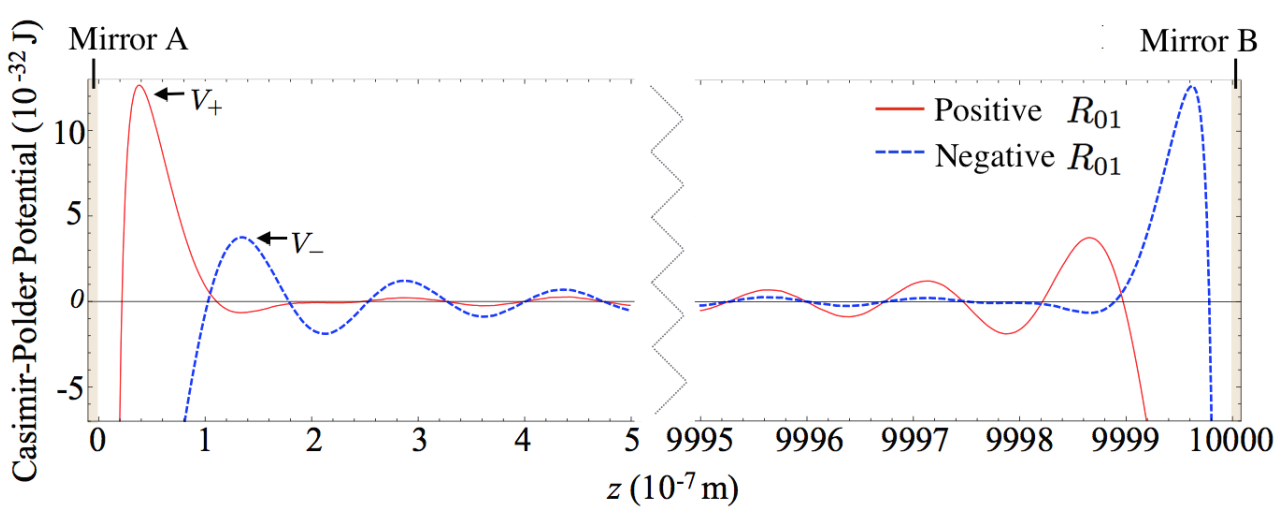

FIG. 5: (Color online) The CP potential experienced by the driven 3-MCP molecule in a superposition of half-excited and half-ground state. Solid red line: 3-MCP molecule with the positive $R_{01}$, dotted blue line: 3 -MCP molecule with the negative $R_{01}$.

pendence on the CP potential. For circularly polarized light, normal mirrors in general reverse its polarization upon reflection, but the chiral mirrors conserve the polarization with positive or negative phase shifts.

\section{A. Electric Circular Dichroism}

The 3-MCP molecules exhibit electric circular dichroism (ECD) by absorbing left- and right-circularly polarized light differently by electric transitions in the ultraviolet region. We have four isomers of 3-MCP molecules with an equatorial (eq) or axial (ax) methyl group in the $(R)-$ or $(S)$-configurations. For the $S_{0} \rightarrow S_{1}$ excitation of the 3 -MCP molecule with an equatorial methyl group, we have the electric transition dipole $\left|\mathbf{d}_{01}\right|=2.44 \times 10^{-31} \mathrm{Cm}$, the optical rotatory strength $\left|R_{01} / c\right|=8.07 \times 10^{-63} \mathrm{C}^{2} \mathrm{~m}^{2}$, and the vertical $S_{0}-S_{1}$ excitation energy $\hbar \omega_{10}=E_{1}-E_{0}=4.24 \mathrm{eV}\left(\omega_{10}=\right.$ $\left.6.44 \times 10^{15} \mathrm{~s}^{-1}\right)$ [34, 35]. Since the optical rotatory strength of the molecule with an equatorial methyl group is slightly larger than that of the molecule with an axial methyl group, we use the molecule with an equatorial methyl group for a quantitative analysis in this article. Since the enantiomers have the optical rotatory strength of opposite sign and equal in magnitude for every transitions, the chiral component of the $\mathrm{CP}$ potential (4) can be used to separate them. Here an $(R)-3-($ eq)-MCP molecule and an $(S)-3-($ eq)-MCP molecule have a positive and negative optical rotatory strength $R_{01}$ respectively. Since the ratio $\left|\mathbf{d}_{01}\right|^{2} /\left|R_{01} / c\right|=7.38$ is relatively small, the chiral component of the potential is comparable to its electric component for the molecules. The average thermal photon number is generally very small for the case of ECD (i.e., $n\left(\omega_{10}\right) \approx 10^{-72}$ for the $S_{0} \rightarrow S_{1}$ excitation of the 3-MCP molecule at room temperature $T=298 \mathrm{~K})$, and the temperature dependence of the $\mathrm{CP}$ potential is usually negligible for the case of ECD.

The molecule in the excited state has the resonant contributions to the CP potential caused by real pho- tons. When the molecule is far away from the mirrors (i.e., $z, a-z>\lambda_{10}=\frac{2 \pi c}{\omega_{10}}$ ), they are generally dominant over the non-resonant contributions from virtual photons. For this reason, we introduced a laser field to drive the molecules to excite them. In FIG. 2, the electric component and the chiral component of the CP potential experienced by $3-\mathrm{MCP}$ molecule near mirror A with the parameters above are plotted respectively with the cavity width $a=1 \mathrm{~mm}$ (Appendix A). The chiral component in the figure is the one acting on the molecule with the positive $R_{01}$ (i.e., $(R)-3$-(eq)-MCP molecule), and it changes sign for the molecule with the negative $R_{01}$ (i.e., $(S)$-3-(eq)-MCP molecule). Since mirror B has opposite chirality from mirror A, the molecule sees the chiral component with opposite sign near mirror B. The magnitude of the electric component and that of the chiral component depend linearly on the reflection coefficient $r_{e}$ and $r_{c}$ respectively. For our purposes, it is desirable to introduce nearly perfect chiral mirrors with $r_{e} \approx 0$ and $r_{c} \approx 1$. For the 3-MCP molecules, the magnitude of the chiral component is similar to that of the electric component when $r_{c}$ is around 10 times as large as $r_{e}$.

In [36], an enhancement of the the electric component of the CP potential was observed when the cavity width $a$ is equal to a half integer multiple of the molecular transition wavelength, i.e., $a=\nu \lambda_{10} / 2, \nu \in \mathbb{N}$. For the chiral component, it is found that such enhancement occurs when the cavity width $a=\nu \lambda_{10} / 4$ where $\nu$ is even/odd natural number when the two mirrors of the cavity have the same/opposite chirality respectively. In FIG. 33, the electric and chiral component of the $\mathrm{CP}$ potential with $r_{e}=0.05, r_{c}=0.8$ and the cavity width $a=\nu \lambda_{10} / 4$ is plotted. It can be seen that the chiral component is enhanced when $\nu$ is odd, i.e., $\nu=7$, while the electric component increases as $a$ becomes close to a half integer multiple of the molecular transition wavelength. However this enhancement can only be seen if we have a very small cavity whose width contains only a few molecular transition wavelengths, and a strong enhancement only occurs when the reflection coefficients are very close to 1 . 
In this article, we choose a realistic setup with $a=$ $1 \mathrm{~mm}$ where such enhancement can not be observed and rather the $\mathrm{CP}$ potential behaves as if it is the sum of two independent potentials created by two mirrors. Then the potential far away from the mirrors is negligible, and it only becomes significant when the molecule is very close to the mirrors as shown in FIG. 2. Therefore our results presented in this article can be used even for the setup with a single chiral mirror, and the cavity setup here is only used for the purpose of constraining the molecular beam inside the cavity.

\section{B. Vibrational Circular Dichrosim}

In contrast to cases of ECD, we often observe the temperature dependence of the CP potential caused by thermal photons in the infrared region when we use molecules with vibrational circular dichroism (VCD). In FIG. 4 the temperature dependence of the CP potential experienced by the $(R)$-propylene-oxide molecule with $\left|\mathbf{d}_{01}\right|=8.82 \times 10^{-32} \mathrm{Cm}, R_{01} / c=3.89 \times 10^{-67} \mathrm{C}^{2} \mathrm{~m}^{2}$ (positive optical rotatory strength), and $\omega_{10}=3.8 \times 10^{13} \mathrm{~s}^{-1}$ 37] is shown as an example. The reflection coefficients are set to be $r_{e}=0.05, r_{c}=0.8$.

Here we have the average thermal photon number $n\left(\omega_{10}\right)=0.6$ at room temperature and the $\mathrm{CP}$ potential increases with temperature. As it can be seen in the figure, the use of molecules with vibrational circular dichroism has the advantage that the peaks of the $\mathrm{CP}$ potential appear far from the mirror due to the long wavelength of photons involved. However it has challenges due to the weakness of the $\mathrm{CP}$ potential (7) which depends on the frequency of photons. Therefore, in the next section, we use the 3-MCP molecules with the stronger CP potential (FIG. 2) in order to analyze our setup to separate enantiomers.

\section{SEPARATION OF ENANTIOMERS}

Next, we study trajectories of the 3-MCP molecules in the cavity. The $\mathrm{CP}$ force can be obtained by $F_{C P}=$ $-d U_{C P} / d z$ where $U_{C P}$ is given in (1). We set $r_{c}=0.8$, $r_{e}=0.05$ and the width of the cavity $a=1 \mathrm{~mm}$. The mass of one 3 -MCP molecule is approximately equal to $1.63 \times 10^{-25} \mathrm{~kg}$. We choose a laser intensity $I=5 \mathrm{~W} / \mathrm{cm}^{2}$, a Rabi frequency $\Omega=2\left|\mathbf{d}_{01}\right|(2 \pi I / c)^{1 / 2} / \hbar=1.42 \times 10^{7} \mathrm{~s}^{-1}$ [38, and the detuning $\Delta=\omega_{L}-\omega_{10}=2 \pi \times 0.1 \mathrm{MHz}$. When $\Delta \leq 0.1 \Omega$, approximately half of the molecules are in the excited state, and the other half are in the ground state. FIG. 5 shows the CP potential experienced by the molecule in such a superposition state. Near mirror $\mathrm{A}$, the molecules with the positive $R_{01}$ and those with the negative $R_{01}$ are subject to a high potential barrier $V_{+}\left(1.26 \times 10^{-31} \mathrm{~J}\right)$ and a low potential barrier $V_{-}\left(3.76 \times 10^{-32} \mathrm{~J}\right)$ respectively. The differences in the potential barriers seen by these enantiomers come from (a)

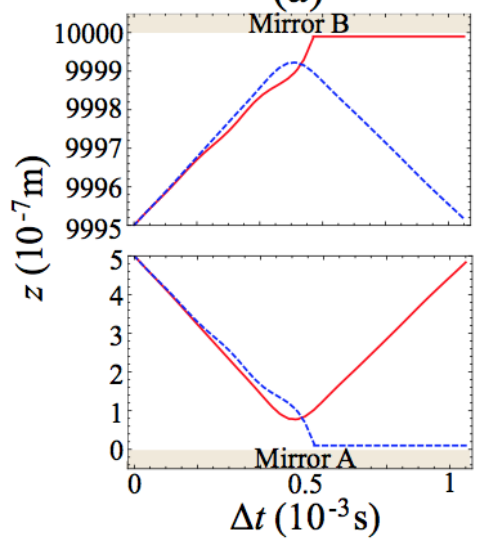

(b)
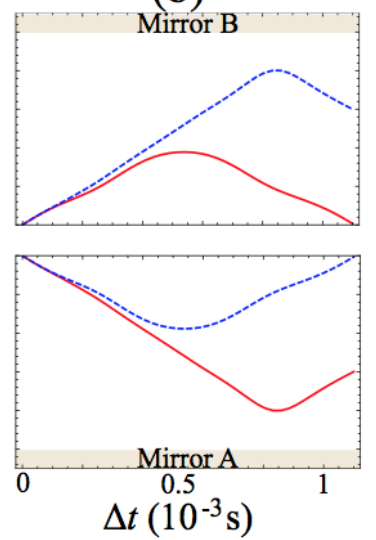

(c)

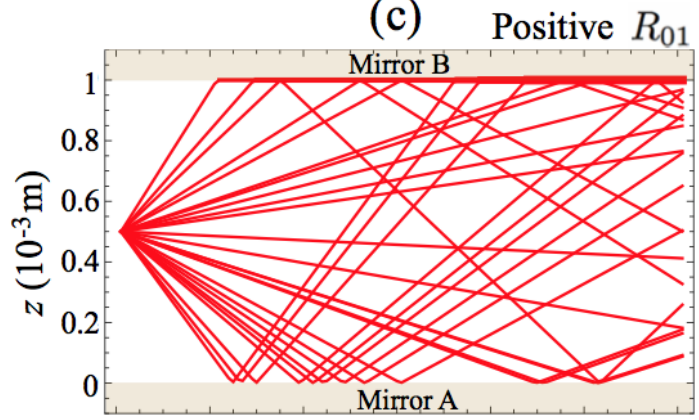

Mirror A

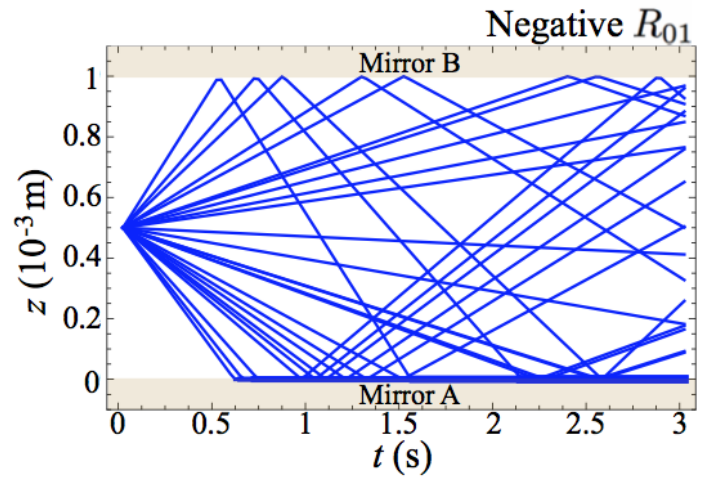

FIG. 6: (Color online) Sample trajectories (near the mirrors $(a, b))$ with different initial velocities. $(a, b)$ Solid red lines are for the molecules with the positive $R_{01}$ and dotted blue lines are for those with the negative $R_{01} \cdot v_{z}=+/-0.9 \mathrm{~mm} / \mathrm{s}$ (above/below) for (a), and $v_{z}=+/-0.5 \mathrm{~mm} / \mathrm{s}$ (above/below) for (b). (c) Trajectories of the molecules with the positive $R_{01}$ (above) and those with the negative $R_{01}$ (below) with Gaussian distributed initial velocities with mean $0 \mathrm{~mm} / \mathrm{s}$ and standard deviation $0.4 \mathrm{~mm} / \mathrm{s}$. (25 trajectories)

the chiral component of the $\mathrm{CP}$ potential, and the behavior of the potential becomes opposite near mirror $\mathrm{B}$ which has the chirality opposite to that of mirror A. $1.26 \times 10^{-31} \mathrm{~J}$ and $3.76 \times 10^{-32} \mathrm{~J}$ correspond to kinetic energy of the molecule with speed $1.2 \mathrm{~mm} / \mathrm{s}$ and $0.7 \mathrm{~mm} / \mathrm{s}$ respectively. Therefore, near mirror $\mathrm{A}$, if the initial speed of the molecule $\left|v_{z}\right|$ in the $\hat{z}$-direction (perpendic- 
ular to the plane of mirrors) is in between $0.7 \mathrm{~mm} / \mathrm{s}$ and $1.2 \mathrm{~mm} / \mathrm{s}$, the molecule with the negative $R_{01}$ climbs up the low potential barrier and gets attracted to mirror A, while the one with the positive $R_{01}$ can not overcome the high potential barrier and gets repelled back to the opposite side of the cavity (FIG. 6 (a, below)). The opposite behavior of the enantiomers can be observed near mirror B (FIG. 6 (a, above)). When $\left|v_{0 z}\right|$ is smaller than $0.7 \mathrm{~mm} / \mathrm{s}$, both enantiomers can not overcome the potential barrier and get reflected back to the center of the cavity (FIG. 6 (b)). In this way, we aim to collect the molecules with the positive $R_{01}$ and those with the negative $R_{01}$ near mirror $\mathrm{B}$ and mirror $\mathrm{A}$ respectively.

We now consider the molecular beam initially injected to the center of the cavity (i.e., initial position of molecule $z_{0}=0.5 \mathrm{~mm}$ ) with distribution for initial velocities in $\hat{z}$ direction being a Gaussian with mean $0 \mathrm{~mm} / \mathrm{s}$ and standard deviation $0.4 \mathrm{~mm} / \mathrm{s}$. The time evolution of positions
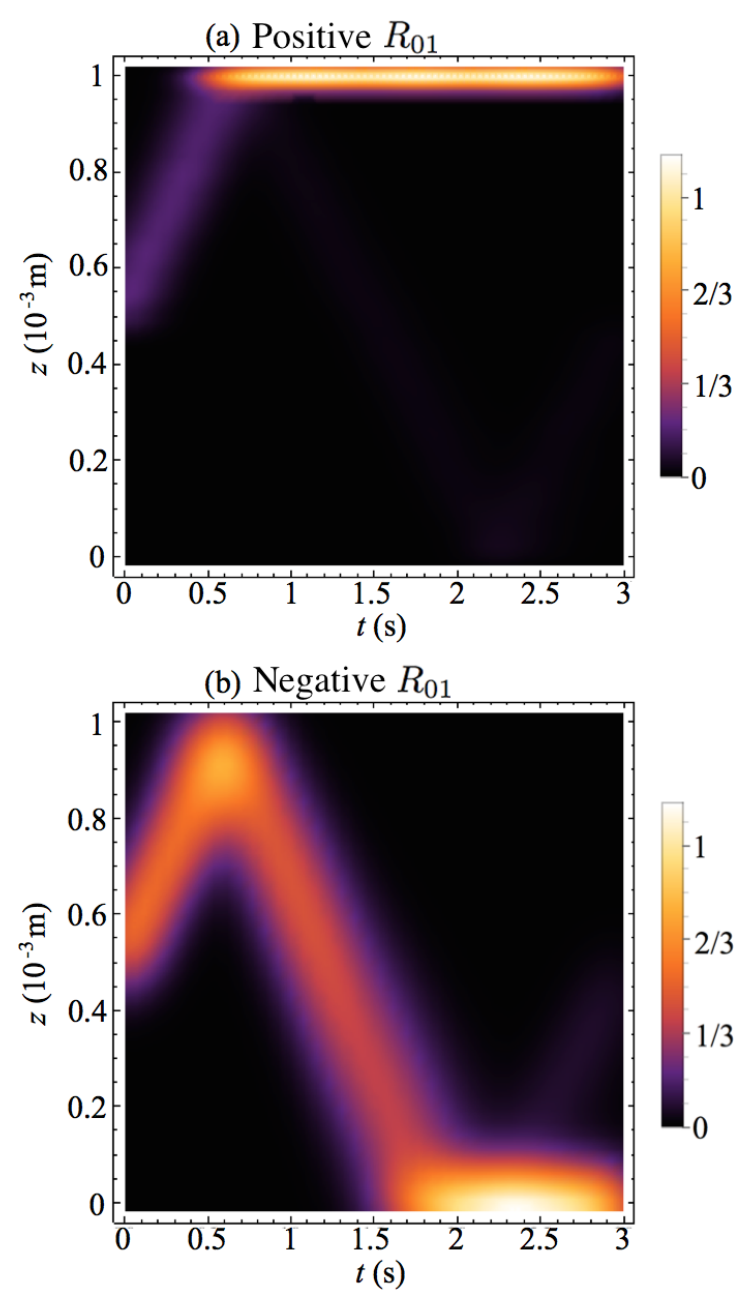

FIG. 7: (Color online) Trajectories of (a) the molecules with the positive $R_{01}$ and (b) those with the negative $R_{01}$ whose initial velocities are Gaussian distributed with mean $0.8 \mathrm{~mm} / \mathrm{s}$ and standard deviation $0.1 \mathrm{~mm} / \mathrm{s}$. (100 trajectories) of molecules can be obtained numerically (FIG. 6 (c)). As can be seen, $\sim 10 \%$ of the molecules with the positive $R_{01}$ and those with the negative $R_{01}$ get collected in the vicinity of mirror $\mathrm{B}$ and of mirror A respectively after $\sim 1$ second.

A more efficient separation can be realized when the molecules have initial velocities toward one of the mirrors. For example, if the distribution for initial velocities is a Gaussian with mean $0.8 \mathrm{~mm} / \mathrm{s}$ and a standard deviation $0.1 \mathrm{~mm} / \mathrm{s}$ so that the molecules move toward mirror B initially, around $90 \%$ of the molecules with the positive $R_{01}$ and those with the negative $R_{01}$ get collected near mirror B and mirror A respectively after $\sim 1.5$ seconds, with the remaining $10 \%$ staying around the center of the cavity (FIG. 7).

\section{CONCLUSION}

To summarise, we have presented a realistic proposal for a Stern-Gerlach type separator for chiral enantiomers based on discriminatory $\mathrm{CP}$ forces. We have shown that the enantiomer-selective excited-state $\mathrm{CP}$ force due to electric molecular dichroism is most suitable for this purpose. It exhibits pronounced repulsive potential barriers when using chiral mirrors which selectively repel only one of the two enantiomers while attracting the other. By sending a molecular beam through a cavity formed by two mirrors of opposite chirality, each enantiomer gets attracted to one mirror and repelled from the other, leading to an efficient separation. Our analysis shows that our setup can be used for separation of enantiomers, as well as for the detection of the chiral dependence of the $\mathrm{CP}$ potential within the reach of current technology. Compared to conventional methods for chiral separations such as chromatography, our method proposed here is more universal since the same chiral mirror can be used for chiral separation of a wide range of molecules.

Two alternatives might be considered in order to potentially improve our scheme. First, while we use an exciting laser parallel to the plates, one could also consider an evanescent laser field emerging from the chiral mirrors themselves. The resulting light force could possibly be designed to enhance chiral effects. Secondly, the use of curved mirrors would lead to a stronger mode confinement and associated enhancement of the CP potential. This could be particularly useful for molecules with vibrational dichroism, where the potential is weak to start with, but cavity enhancement can be made particularly strong as the respective transitions are in the microwave regime.

\section{ACKNOWLEDGMENTS}

We would like to thank P. Barcellona, R. Bennett, S. Fuchs and A. Salam for discussions. This work was supported by the German Research Council (grants 
BU1803/3-1 and GRK 2079/1), a National Science and Engineering Research Discovery Grant in Canada, funds from Canada Foundation for Innovation for the Centre for Research on Ultra-Cold Systems (CRUCS) and Chi- rality Research on Origins and Separation (CHIROS) at UBC. F. S. thanks DAAD Research Grants for support. S.Y.B is grateful for support by the Freiburg Institute of Advanced Studies.
[1] Y. Saito and H. Hyuga, Rev. Mod. Phys. 85, 603-621 (2013).

[2] T. J. Ward and K. D. Ward, Anal. Chem. 84, 626 (2012).

[3] Y. Wang and A. M. Chen, Org. Process Res. Dev. 12, 282-290 (2008).

[4] Y. Li, C. Bruder and C. P. Sun, Phys. Rev. Lett. 99, 130403 (2007).

[5] X. Li and M. Shapiro, J. Chem. Phys. 132, 194315 (2010).

[6] A. Jacob and K. Hornberger, J. Chem. Phys. 137, 044313 (2012).

[7] H. B. G. Casimir and D. Polder, Phys. Rev. 73, 360 (1948).

[8] J. M. Wylie and J. E. Sipe, Phys. Rev. A 32, 2030 (1985).

[9] M. Fichet, F. Schuller, D. Bloch and M. Ducloy, Phys. Rev. A 51, 1553 (1995).

[10] H. Failache, S. Saltiel, M. Fichet, D. Bloch and M. Ducloy, Phys. Rev. Lett. 83, 5467 (1999).

[11] P. Bushev, A. Wilson, J. Eschner, C. Raab, F. SchmidtKaler, C. Becher and R. Blatt, Phys. Rev. Lett. 92, 223602 (2004).

[12] T. H. Boyer, Phys. Rev. A 9, 2078 (1974).

[13] Y. Tikochinsky and L. Spruch, Phys. Rev. A 48, 4236 (1993).

[14] S. Y. Buhmann, D.-G. Welsch and T. Kampf, Phys. Rev. A 72, 032112 (2005).

[15] H. Haakh, F. Intravaia, C. Henkel, S. Spagnolo, R. Passante, B. Power and F. Sols, Phys. Rev. A 80, 062905 (2009).

[16] S. Fuchs, J. A. Crosse and S. Y. Buhmann, Phys. Rev. A 95, 023805 (2017).

[17] D. T. Butcher, S. Y. Buhmann and S. Scheel, New J. Phys. 14, 113013 (2012).

[18] L. Barron, Molecular Light Scattering and Optical Activity (Cambridge, 2004).

[19] C. I. Sukenik, M. G. Boshier, D. Cho, V. Sandoghdar and E. A. Hinds, Phys. Rev. Lett. 70, 560 (1993).

[20] G. Feinberg, Trans. N. Y. Acad. Sci. 38, 26 (1977).

[21] O. L. Zhizhimov and I. B. Khriplovich, ZhETF 82, 1026 (Sov. Phys. JETP, 55, 601) (1982).

[22] I. B. Khriplovich, Parity Non-Conservation in Atomic Phenomena (Gordon and Breach, New York, 1991).

[23] J. K. Jenkins, A. Salam and T. Thirunamachandran, Phys. Rev. A 50, 4767 (1994).

[24] P. Barcellona, H. Safari, A. Salam and S. Y. Buhmann, Phys. Rev. Lett. 118, 193401 (2017).

[25] S. Y. Buhmann, Dispersion Forces I: Macroscopic Quantum Electrodynamics and Ground-State Casimir, Casimir-Polder and van der Waals Forces (Springer, 2012).

[26] S. Y. Buhmann, Many-Body Effects, Excited Atoms, Finite Temperature and Quantum Friction (Springer, 2012).

[27] S. Fuchs, R. Bennett, R. V. Krems and S. Y. Buhmann, Phys. Rev. Lett. 121, 083603 (2018).
[28] S. Fuchs, R. Bennett and S. Y. Buhmann, Phys. Rev. A 98, 022514 (2018).

[29] S. Y. Buhmann and S. Scheel, Phys. Rev. Lett. 100(25), 253201 (2008).

[30] S. Scheel and S. Y. Buhmann, Acta Phys. Slovaca 58(5), 675 (2008).

[31] S. Y. Buhmann and S. Scheel, Phys. Scripta T. 135, 014013 (2008).

[32] T. Matsubara, Prog. Theor. Phys. 14(4), 351 (1955).

[33] S. M. Ali, T. M. Habashy and J. A. Kong, J. Opt. Soc. Am. A 9, 413 (1992).

[34] D. Kröner, J. Phys. Chem. A 115, 14510-14518 (2011).

[35] D. Kröner, Phys. Chem. Chem. Phys. 17, 19643 (2015).

[36] S. Å. Ellingsen, S. Y. Buhmann and S. Scheel, Phys. Rev. A. 80, 022901 (2009).

[37] A. Scherrer, F. Agostini, D. Sebastiani, E. K. U. Gross and R. Vuilleumier, J. Chem. Phys. 143, 074106 (2015).

[38] Y. B. Band, Light and Matter: Electromagnetism, Optics, Spectroscopy and Lasers (Wiley, 2006).

[39] H. C. Chen, Theory of Electromagnetic Waves: A Coordinate-Free Approach (New York: McGraw-Hill, 1983).

\section{Appendix A: Derivation of the Casimir-Polder Potential}

Here we derive the electric and the chiral components of the Casimir-Polder (CP) potential experienced by a molecule in a planar cavity.

Taking the trace of the Green's function is equivalent to taking the dot product between the dyads where

$$
\mathbf{e}_{s} \cdot \mathbf{e}_{p}\left(-k^{\perp}\right)=\mathbf{e}_{p}\left(k^{\perp}\right) \cdot \mathbf{e}_{s}=0
$$

and

$$
\mathbf{e}_{s} \cdot \mathbf{e}_{s}=1, \quad \mathbf{e}_{p}\left(k^{\perp}\right) \cdot \mathbf{e}_{p}\left(-k^{\perp}\right)=-1+\frac{2 k^{\| 2} c^{2}}{\omega^{2}} .
$$

Since $\int d^{2} k^{\|}=2 \pi \int_{0}^{\infty} k^{\|} d k^{\|}$, we have

$\operatorname{tr} \mathbf{G}(\mathbf{r}, \mathbf{r}, \omega)=\frac{i}{4 \pi} \int \frac{d k^{\|} k^{\|}}{k^{\perp}}$

$\times\left[e^{2 i k^{\perp} z}\left(\left(\mathbf{D}^{-1} \cdot \mathbf{R}\right)^{(s, s)}-\left(1-\frac{2 k^{\| 2} c^{2}}{\omega^{2}}\right)\left(\mathbf{D}^{-1} \cdot \mathbf{R}\right)^{(p, p)}\right)\right.$

$+e^{2 i k^{\perp}(a-z)}$

$\left.\times\left(\left(\mathbf{R}^{\prime} \cdot \mathbf{D}^{-1}\right)^{(s, s)}-\left(1-\frac{2 k^{\| 2} c^{2}}{\omega^{2}}\right)\left(\mathbf{R}^{\prime} \cdot \mathbf{D}^{-1}\right)^{(p, p)}\right)\right]$

where we discarded the terms that are independent of the position of the molecule $z$ since we are eventually interested in the CP force given by $F_{C P}=-d U_{C P} / d z$. 
When we introduce $\omega=i \xi$ and $k^{\perp}=i \kappa^{\perp}\left(\kappa^{\perp}=\right.$ and $\left.\sqrt{\xi^{2} / c^{2}+k^{\| 2}}\right)$, we have $\int_{0}^{\infty} d k^{\|} k^{\|} / \kappa^{\perp}=\int_{\xi / c}^{\infty} d \kappa^{\perp}$ and

$\operatorname{tr} \mathbf{G}(\mathbf{r}, \mathbf{r}, i \xi)=\frac{1}{4 \pi} \int_{\xi / c}^{\infty} d \kappa^{\perp}$

$\times\left[e^{-2 \kappa^{\perp} z}\left(\left(\mathbf{D}^{-1} \cdot \mathbf{R}\right)^{(s, s)}+\left(1-\frac{2 \kappa^{\perp 2} c^{2}}{\xi^{2}}\right)\left(\mathbf{D}^{-1} \cdot \mathbf{R}\right)^{(p, p)}\right) \times\left[e^{-2 \kappa^{\perp} z}\left(\left(\mathbf{D}^{-1} \cdot \mathbf{R}\right)^{(s, p)}\left(\frac{2 \kappa^{\perp 2} c^{2}}{\xi^{2}}-1\right)+\left(\mathbf{D}^{-1} \cdot \mathbf{R}\right)^{(p, s)}\right)\right.\right.$
$+e^{-2 \kappa^{\perp}(a-z)}$

$\left.\times\left(\left(\mathbf{R}^{\prime} \cdot \mathbf{D}^{-1}\right)^{(s, s)}+\left(1-\frac{2 \kappa^{\perp 2} c^{2}}{\xi^{2}}\right)\left(\mathbf{R}^{\prime} \cdot \mathbf{D}^{-1}\right)^{(p, p)}\right)\right] \cdot(\mathrm{A} 4)+e^{-2 \kappa^{\perp}(a-z)}\left(\left(\mathbf{R}^{\prime} \cdot \mathbf{D}^{-1}\right)^{(s, p)}\left(\frac{2 \kappa^{\perp 2} c^{2}-1}{\xi^{2}}\right)\right.$

Therefore,

$$
\left.\left.+\left(\mathbf{R}^{\prime} \cdot \mathbf{D}^{-1}\right)^{(p, s)}\right)\right]
$$

$U_{0 e}=\frac{\hbar \mu_{0}}{8 \pi^{2}} \int_{0}^{\infty} d \xi \xi^{2} \alpha(i \xi) \int_{\xi / c}^{\infty} d \kappa^{\perp}$

$\times\left[e^{-2 \kappa^{\perp} z}\left(\left(\mathbf{D}^{-1} \cdot \mathbf{R}\right)^{(s, s)}+\left(1-\frac{2 \kappa^{\perp 2} c^{2}}{\xi^{2}}\right)\left(\mathbf{D}^{-1} \cdot \mathbf{R}\right)^{(p, p)}\right) \quad\right.$ Finally we obtain

$+e^{-2 \kappa^{\perp}(a-z)}$

$\left.\times\left(\left(\mathbf{R}^{\prime} \cdot \mathbf{D}^{-1}\right)^{(s, s)}+\left(1-\frac{2 \kappa^{\perp 2} c^{2}}{\xi^{2}}\right)\left(\mathbf{R}^{\prime} \cdot \mathbf{D}^{-1}\right)^{(p, p)}\right)\right]$

and

$U_{1 e}=-U_{0 e}$

$-\frac{\mu_{0}}{12 \pi} \omega_{10}^{2}\left|\mathbf{d}_{01}\right|^{2} \operatorname{Re}\left\{i \int_{0}^{\infty} \frac{d k^{\|} k^{\|}}{k^{\perp}}\right.$

$U_{0 c}=\frac{\hbar \mu_{0}}{4 \pi^{2} c} \int_{0}^{\infty} d \xi \xi^{2} \Gamma(i \xi) \int_{\xi / c}^{\infty} d \kappa^{\perp}$

$\times\left[e^{2 i k^{\perp} z}\left(\left(\mathbf{D}^{-1} \cdot \mathbf{R}\right)^{(s, s)}-\left(1-\frac{2 k^{\| 2} c^{2}}{\omega_{10}^{2}}\right)\left(\mathbf{D}^{-1} \cdot \mathbf{R}\right)^{(p, p)}\right)\right.$

$\times\left[e^{-2 \kappa^{\perp} z}\left(\left(\mathbf{D}^{-1} \cdot \mathbf{R}\right)^{(s, p)}\left(\frac{2 \kappa^{\perp 2} c^{2}}{\xi^{2}}-1\right)+\left(\mathbf{D}^{-1} \cdot \mathbf{R}\right)^{(p, s)}\right)\right.$

$+e^{-2 \kappa^{\perp}(a-z)}\left(\left(\mathbf{R}^{\prime} \cdot \mathbf{D}^{-1}\right)^{(s, p)}\left(\frac{2 \kappa^{\perp 2} c^{2}-1}{\xi^{2}}\right)\right.$

$+e^{2 i k^{\perp}(a-z)}$

$\left.\left.\times\left(\left(\mathbf{R}^{\prime} \cdot \mathbf{D}^{-1}\right)^{(s, s)}-\left(1-\frac{2 k^{\| 2} c^{2}}{\omega_{10}^{2}}\right)\left(\mathbf{R}^{\prime} \cdot \mathbf{D}^{-1}\right)^{(p, p)}\right)\right]\right\}$.

and

For the chiral components of the potential, we use $\mathbf{a} \times$ $\mathbf{b c}=(\mathbf{a} \times \mathbf{b}) \mathbf{c}$ 39], then

$$
\nabla \times \mathbf{e}_{s}=-i \frac{\omega}{c} \mathbf{e}_{p}\left(k^{\perp}\right), \quad \nabla \times \mathbf{e}_{p}\left(k^{\perp}\right)=i \frac{\omega}{c} \mathbf{e}_{s} .
$$

Therefore,

$$
\begin{aligned}
& \operatorname{tr}[\nabla \times \mathbf{G}(\mathbf{r}, \mathbf{r}, \omega)]=\frac{\omega}{4 \pi c} \int \frac{d k^{\|} k^{\|}}{k^{\perp}} \\
& \times\left[e^{2 i k^{\perp} z}\left(\left(\mathbf{D}^{-1} \cdot \mathbf{R}\right)^{(s, p)}\left(\frac{2 k^{\| 2} c^{2}}{\omega^{2}}-1\right)-\left(\mathbf{D}^{-1} \cdot \mathbf{R}\right)^{(p, s)}\right)\right. \\
& +e^{2 i k^{\perp}(a-z)}\left(\left(\mathbf{R}^{\prime} \cdot \mathbf{D}^{-1}\right)^{(s, p)}\left(\frac{2 k^{\| 2} c^{2}}{\omega^{2}}-1\right)\right. \\
& \left.\left.-\left(\mathbf{R}^{\prime} \cdot \mathbf{D}^{-1}\right)^{(p, s)}\right)\right], \quad \text { (A8) }
\end{aligned}
$$

$U_{1 c}=-U_{0 c}$

$+\frac{\mu_{0} \omega_{10}^{2} R_{01}}{6 \pi c} \operatorname{Re}\left\{\int \frac{d k^{\|} k^{\|}}{k^{\perp}}\right.$

$\times\left[e^{2 i k^{\perp} z}\left(\left(\mathbf{D}^{-1} \cdot \mathbf{R}\right)^{(s, p)}\left(\frac{2 k^{\| 2} c^{2}}{\omega_{10}^{2}}-1\right)-\left(\mathbf{D}^{-1} \cdot \mathbf{R}\right)^{(p, s)}\right)\right.$

$+e^{2 i k^{\perp}(a-z)}\left(\left(\mathbf{R}^{\prime} \cdot \mathbf{D}^{-1}\right)^{(s, p)}\left(\frac{2 k^{\| 12} c^{2}}{\omega_{10}^{2}}-1\right)\right.$

$\left.\left.\left.-\left(\mathbf{R}^{\prime} \cdot \mathbf{D}^{-1}\right)^{(p, s)}\right)\right]\right\}$. 